\title{
Pathological root resorption as a possible intrusive injury of a deciduous mandibular second molar - A unique clinical vignette
}

\author{
Ghousia $\mathbf{S}^{1^{*}}$, Nyer Firdoose $\mathbf{C ~ S}^{2}$ \\ ${ }^{1}$ Consultant Pediatric Dentist, ${ }^{2}$ Clinical Director \& Head, Oral Maxillofacial Surgeon, Dental Health Care \& Research Unit @ Gharonda, \\ Bangalore, Karnataka, India \\ *Corresponding Author: Ghousia S \\ Email: drghousia786@gmail.com
}

\begin{abstract}
A primary teeth and its permanent tooth germ are most of the time seen as an inter-dependent units, where each one of them interacts with and depends on each other. Intrusive injury is a most common type of an injury to primary dentition and requires a pediatric dentist to carefully examine not only the damaged tooth, but possible sequelae to the permanent tooth germ.

Alteration in eruption and / or in development, as a consequence of inflammation / infection of the preceding primary teeth, such as: hypoplasia, morphological alteration on the dental crown or total arrest of radicular formation. The response of the dento-alveolar apparatus to infection is characterized by inflammation which may result in tooth resorption. This may be a consequence of infective endodontic pathosis alone or superimposed on trauma induced resorption. These infection induced resoprtions, which are generally termed inflammatory root resorptions, may occur as internal resorptions, external resorptions or combined internal - external lesions.

This manuscript will present a rare case of a primary tooth onto its permanent tooth bud that showed one such case scenario of a pathological root resorption of primary teeth following a posssible intrusive injury. The condition analysed in this study belong to a patient who visited our Children's Dentistry Clinic. These conditions are often overlooked by parents mainly because less attention is given to the primary dentition and to the child's inability to cope with the situation. The earlier this kind of condition is diagnosed, the less would be the destructive effects and the consequences on the permanent germ unit.
\end{abstract}

Keywords: Anatomic proximity, Intrusive injury, Inflammatory root resorption, Pathological root resorption, Space regainer.

\section{Introduction}

Owing to the anatomic proximity between the root of the primary tooth and its permanent successor, trauma to primary dentition may cause developmental disturbances in unerupted permanent teeth. Primary teeth are more vulnerable to intrusive dental injuries because of large trabecular spaces which is an inherent characteristic of the developing bone tissue around primary teeth and the presence of root resorption. Resilience of the alveolar bone in young children causes dental luxations of the intrusive type to be more common than crown fractures (Bennett, 1964; Taintor et al., 1979; Joho and Marechaux, 1980). An intrusive injury is the consequence of impact by a force in an axial direction that results in displacement of the tooth within the socket. In addition, the short roots, resorbing roots, and high crown-root ratio of primary teeth offer less resistance to intrusive displacement (Von Arx, 1993). Displacement of the primary tooth root may affect development of the permanent tooth by altering the secretary phase of the ameloblasts resulting in enamel hypoplasia or, in subsequent stages, changing the root formation process.

Inflammatory root resorption in primary teeth is a frequent finding in the clinical practice of pediatric dentists. ${ }^{1}$ Radiographically, inflammatory root resorption is characterized by the gradual loss of tooth substance associated with persistent, progressive radiolucency of the adjacent alveolar bone. ${ }^{2,3}$ This leads to an imbalance in the stomatognathic system and problems such as unerupted permanent successors and the early loss of primary teeth. ${ }^{1,3-}$ 10
Inflammatory root resorption is generally diagnosed by clinical and radiological examinations, especially periapical radiographs. ${ }^{11,12}$ This type of resorption is a consequence and/or complication of different clinical situations, such as pulp, periodontal and/or periradicular infectious processes, dental injury, orthodontic forces, or excessive occlusal forces. ${ }^{1,6}$

Anatomical aspects peculiar to the primary teeth, such as reduced enamel and dentin thickness, greater permeability and lower hardness and strength, further contribute to the more rapid spread of infectious processes in the pulp tissue, which can trigger an inflammatory process and root resorption. ${ }^{13,14}$ Treatment consists of removing the triggering factors, whose determination is critical to choosing the most appropriate protocol. ${ }^{9}$

\section{Case Presentation}

Two girl patients (Twin 1 and Twin 2), aged 5.8 years with a non-contributory medical history presented to our dental operatoty. The twins were non-identical.

One of the girl child (Twin 1) presented with a chief complaint of pain in the lower left back teeth region. On clinical examination the chid revealed class II dental caries w.r.t 74. On radiographic examination, 74 showed external root resorption with internal infection. It was a combination of internal and external root resorption. Treatment plan was to extract 74 followed by a band and loop space maintainer.

The other girl child (Twin 2) presented with a chief complaint of tooth missing in the lower right back teeth region. On clinical examination there was an edentulous space w.r.t 85 , followed by loss of space in the same area 
due to mesial migration of 46 and distal migration of 84 . The child revealed no history of tooth extraction till date. An intra-oral radiograph was taken initially to rule out any congenital missing of 85 .

\section{Investigations}

Intra-oral periapical radiograph revealed a hard tissue over the tooth bud of 45 . To obtain a clear picture, OPG was further suggested which revelaed the 85 being sunken into the alveolus and encroaching the space and position of tooth bud of 45. The teeth present were except 51, 61. This probably happened due to the chewing or occlusal forces of the child on the tooth which must have got external and internal resorption of the roots of 85 as seen in the case of Twin 1.

\section{Treatment}

Treatment was planned for surgically exposing the space and removing the submerged 85 from within the alveolus to prevent further danger to the tooth bud of 45 . The limitations of the surgery being the space between 46 and 84 being very little, nevertheless we decided to take an occlusal approach for the incision and extend the flap to buccal so as to allow access to crown of 85 . The whole proceedure involved a lot of behaviour management skills and control of bleeding into the area and not to hasten in removing or extracting any hard tissue without being sure if the grabbed hard tissue was crown of 85 or tooth bud of 45 .

The crown on 85 was tilted and moved in horizontal direction, as the mesio-distal of 85 length was bigger than the area between 46 and 84 . Post-operatively, we decided to go for a space regainer with a slight modification of Band and Loop with active helical loop incorporation within the loop.

\section{Out-come and Follow Up}

The space difference to be achieved by $7 \mathrm{~mm}$ as the mesiodistal width of 45 tooth bud is $12 \mathrm{~mm}$. So far we have gradually achieved $3 \mathrm{~mm}$. With subsequent activation and follow ups the succedaneous tooth comfortably in line with occlusion in the arch along with the neighbouring teeth as observed with 2 yrs follow-up.

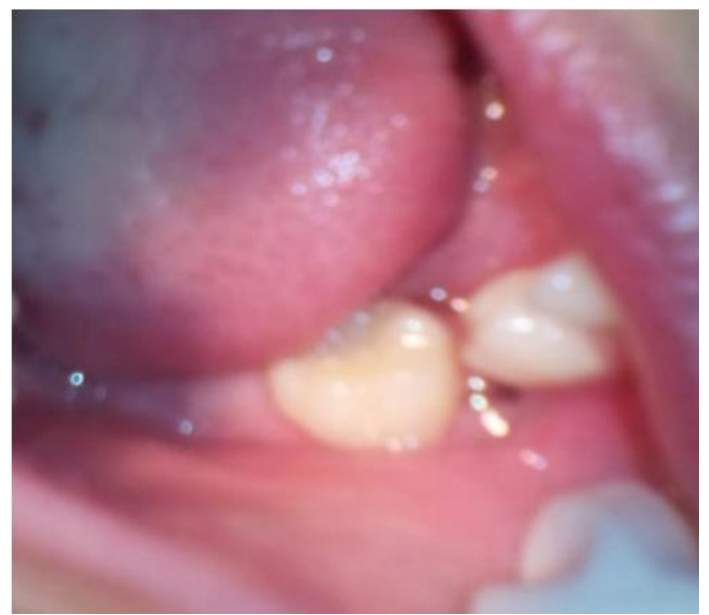

Fig. 1

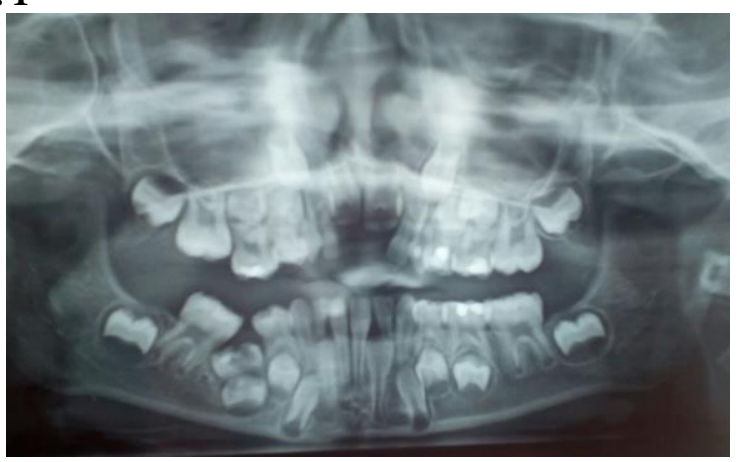

Fig. 2

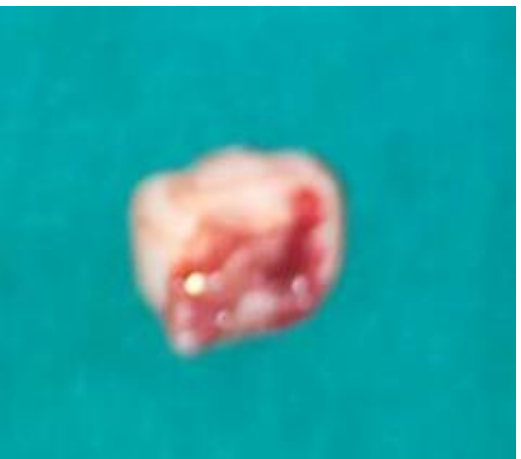

Fig. 3

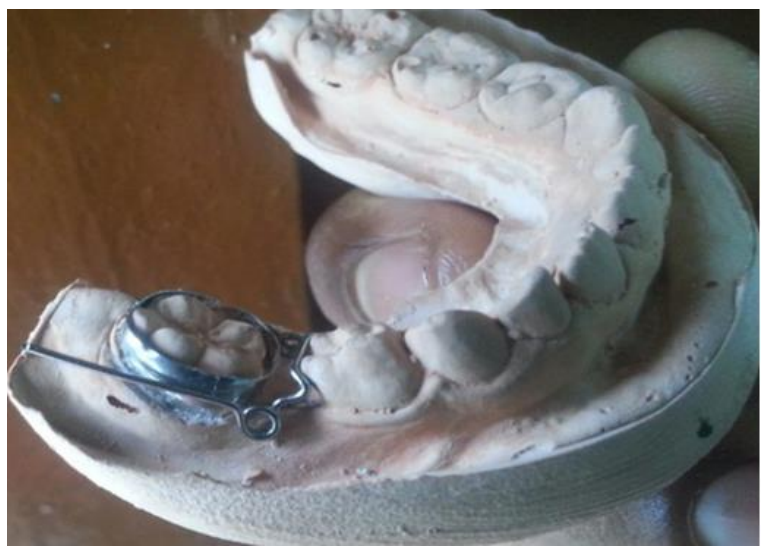

Fig. 4

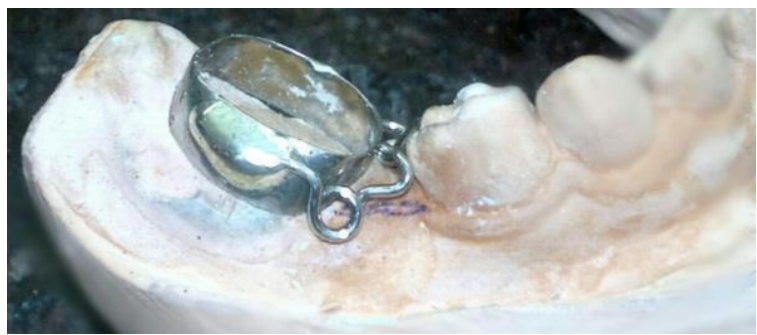

Fig. 5

\section{Discussion}

The major concern with primary tooth trauma is the potential for damage to the underlying permanent successor. ${ }^{1}$ This may occur directly from the injury or from the residual infection associated with the traumatized 
primary tooth. ${ }^{2}$ The topographic relationship of the primary teeth to the permanent tooth germs explains the potential for possible developmental disturbances. Several developmental anomalies have been reported in the permanent dentition as sequelae of injuries to their primary predecessors. ${ }^{2-4}$ These developmental defects may be simple or complex, extensive or local, affecting the crown, root, or the entire germ. In the coronal region, structural alterations may occur such as enamel hypoplasia, crown dilaceration and white, yellow or brown discoloration. Disturbances affecting the root region include root duplication, root dilaceration and partial or complete arrest of root formation. When the entire permanent tooth germ is affected, the following may be noted: alterations to the process of eruption of the permanent tooth, retention of the permanent tooth or malformation of the permanent tooth germ giving the appearance of an odontoma. Do Espírito Santo Jácomo DR et al. ${ }^{5}$ in their longitudinal study of eight years reported that the most common developmental disturbances were discoloration of enamel and/or enamel hypoplasia (46.08\%) and eruption disturbances (17.97\%) due to the traumatic injury in their predecessors. The extent of the disturbance of the developing tooth germ is related to the stage of germ development (the child's age at the time of injury), the spatial relationship of the involved teeth, the type of trauma, the severity, and the direction of impact. ${ }^{6-9}$ Intrusion injuries which constitute $4.4-22 \%$ of traumatic injuries in the primary dentition have been reported to be associated with the highest likelihood of damage to the underlying permanent teeth. ${ }^{1,3,10-15}$

The degree of intrusion can be divided into 3 grades (Von Arx, 1995)

1. Grade I. Mild partial intrusion in which more than $50 \%$ of the crown is visible.

2. Grade II. Moderate partial intrusion in which less than $50 \%$ of the crown is visible.

3. Grade III. Severe or complete intrusion of the crown

According to the Glossary of the American Association of Endodontists, resorption is defined as a condition associated with either a physiologic or a pathologic process resulting in loss of dentin, cementum or bone. ${ }^{1}$ Physiologic resorption is seen in primary teeth that results in their exfoliation and allows eruption of their permanent successors. ${ }^{2,3}$ Pathologic resorption can occur following traumatic injuries, orthodontic tooth movement, or chronic infections of the pulp or periodontal structures. ${ }^{1}$ Pathologic resorption if untreated will result in the premature loss of the affected teeth. ${ }^{4}$

Root resorption may be classified based on its location in relation to the root surface: Internal or external. ${ }^{3}$ Internal resorption may be classified as internal inflammatory resorption and internal replacement resorption. External resorption is further classified into external surface resorption, external inflammatory resorption, external replacement resorption, external cervical resorption and transient apical breakdown. ${ }^{1,3}$

This case report exemplifies inflammatory internal resorption. For internal resorption to progress, the pulp tissue apical to the resorptive lesion must have viable blood supply that provides clastic cells whereas the infected necrotic coronal pulp tissue provides stimulation for those clastic cells. ${ }^{1,4}$ Bacteria probably entered the pulp space through the fracture site thus infecting the coronal pulp tissue which served as a stimulus for the resorption process resulting in the damage to the odontoblast layer and predentin. Wedenberg and Lindskog reported that damage to both these layers results in exposure of the underlying mineralized dentin to odontoclasts. ${ }^{12}$ The resorptive defect that forms is filled with inflammatory connective tissue. Failure to intervene at this stage could result in further progress of the resorption in the defect laterally resulting in a perforation or the resorption may progress apically and the pulp tissue apical to the resorptive defect may undergo necrosis. ${ }^{13}$

This type of resorption results from a low-grade irritation of pulpal tissues such as chronic irreversible pulpitis or partial necrosis that is usually localized to a small area of the root canal system. ${ }^{34}$ The resorption process starts once the odontoblast layer and predentin are disrupted. ${ }^{1,35}$ Metaplastic tissue could resemble bone or cementum. ${ }^{36}$ Though teeth with internal replacement resorption displaying metaplastic changes often present a challenge to the clinician, management is not always impossible.

\section{Conclusion}

Difficulty in gaining compliance from a very young child and the risk of damaging the permanent tooth germ makes the management of these injuries challenging. Careful clinical and radiographic examination along with regular follow-up is essential.

\section{Source of funding}

None.

\section{Conflict of interest}

None.

\section{References}

1. Cordeiro MM, Rocha MJ. The effects of periradicular inflamation and infection on a primary tooth and permanent successor. J Clin Pediatr Dent 2005 Spring;29(3):193-200.

2. Ceyhun A, Zafer CC, Gunseli G, Cengizan A, Ankara. Traumatic intrusion of primary teeth and its effects on the permanent successors: A clinical follow-up study. Oral Surg, Oral Med, Oral Pathol, Oral Radiol, Endodontol 2009;107(4):493-8.

3. Rajni N and Naveen M. Sequelae of Intrusive Trauma to the Primary Predecessors: Odontoma Like Malformation and Enamel Hypoplasia. Austin J Dent 2014;1(1):1005.

4. Raquel Gonçalves Vieira-Andrade, Clarissa Lopes Drumond, Laura Pereira Azevedo Alves, Leandro Silva Marques, Maria Letícia Ramos-Jorge. Inflammatory root resorption in primary molars: prevalence and associated factors. Braz Oral Res 2012;26(4)

5. Marina Fernandes, Ida de Ataide, and Rahul Wagle. Tooth resorption part I - pathogenesis and case series of internal resorption. J Conserv Dent 2013;16(1):4-8.

6. GS Heithersay. Management of tooth resorption. Aust Dent J Suppl 2007;52;(1 Suppl):S105-S21. 
7. Lisa Germain. Tooth resorption: The "Black Hole" of dentistry. Dentistry Today; 34;12:78.

8. R G V Andrade, C L Drumond, L P A Alves, L S Marques, M L Ramos-Jorge. Inflammatory root resorption in primary molars: prevalence and associated factors. J Oral Sci 2007;49(1):1-12.

9. Megha Gupta. Intrusive luxation in primary teeth - Review of literature and report of a case. Saudi Dent J 2011; 23(4):16776.

10. D P Mulia, I S Indiarti, S B Budiarjo. Effect of root resorption of primary teeth on the development of its permanent successors: An evaluation of panoramic radiographs in 7-8 year-old boys. IOP Conf. Series: J Physics Conf Series 1073 (2018) 032015 doi :10.1088/1742-6596/1073/3/032015

11. Andreasen FM, Andreasen JO. Diagnosis of luxation injuries: the importance of standardized clinical, radiographic and photographic techniques in clinical investigations. Endod Dent Traumatol 1985;1(5):160-9.

12. Sameshima GT, Asgarifar KO. Assessment of root resorption and root shape: periapical vs panoramic films. Angle Orthod 2001;71(3):185-9.

13. Morabito A, Defabianis P. A SEM investigation on pulpalperiodontal connections in primary teeth. ASDC J Dent Child 1992:59(1):53-7.

14. Ne RF, Witherspoon DE, Gutmann JL. Tooth resorption. Quintessence Int 1999;30(1):9-25.

How to cite this article: Ghousia S, Firdoose NCS. Pathological root resorption as a possible intrusive injury of a deciduous mandibular second molar - A unique clinical vignette. Int J Oral Health Dent 2019;5(4):216-9. 